\title{
Proteome Analyses of Hepatocellular Carcinoma
}

\author{
Dominik A. Megger ${ }^{5 *}$, Wael Naboulsi ${ }^{51}$, Helmut E. Meyer ${ }^{1,2}$ and Barbara Sitek ${ }^{* 1}$ \\ ${ }^{1}$ Medizinisches Proteom-Center, Ruhr-Universität Bochum, Bochum, Germany; ${ }^{2}$ Leibniz-Institut für Analytische Wissenschaften - \\ ISAS - e.V., Dortmund, Germany
}

\begin{abstract}
Proteomics has evolved into a powerful and widely used bioanalytical technique in the study of cancer, especially hepatocellular carcinoma (HCC). In this review, we provide an up to date overview of feasible proteome-analytical techniques for clinical questions. In addition, we present a broad summary of proteomic studies of HCC utilizing various technical approaches for the analysis of samples derived from diverse sources like HCC cell lines, animal models, human tissue and body fluids.

(C) 2014 The Second Affiliated Hospital of Chongqing Medical University. Published by XIA \& HE Publishing Ltd. All rights reserved.
\end{abstract}

\section{Introduction}

Liver diseases are a major cause of morbidity and mortality worldwide. Particularly hepatocellular carcinoma (HCC) is the fifth most common malignancy worldwide, and its incidence is increasing. Successful therapy for HCC patients with a concomitant increase in survival rate strongly depends on the time and accuracy of diagnosis. Therefore, biomarkers for early and accurate diagnosis of HCC using either body fluid

Keywords: Proteomics; Quantitative analysis; Hepatocellular carcinoma; Biomarker; Cell lines; Animal models; Tissue samples.

Abbreviations: 2D-DIGE, two-dimensional differential gel electrophoresis; AFP, alpha-fetoprotein; ANLN, actin-binding protein anillin; AOX, acyl-COA oxidase; ARLPs, aldose reductase-like proteins; BHMT, betaine-homocysteine S-methyltransferase 1 ; CAPN4, calpain small subunit 1 ; cICAT, cleavable isotope-coded affinity tags; EB1, APC-binding protein EB1; ECM, extracellular matrix; ESI-MS/ MS, electrospray-ionization mass spectrometry; FLNB, filamin-B; HBV, hepatitis virus $B$; HBX, hepatitis B virus X protein; HCC, hepatocellular carcinoma; HSP 27, heat-shock protein 27; hTERT, human telomerase reverse transcriptase; Ig, immunoglobulin; LC, liver cirrhosis; iTRAQ, isobaric tags for relative and absolute quantification; LCM, laser capture microdissection; LC-MS/MS, liquid chromatography coupled tandem mass spectrometry; LT, liver transplantation; MALDI-TOF MS, matrix-assisted laser desorption-ionization time-of-flight mass spectrometry; MCs, microcystins; MRM, multiple reaction monitoring; MS, mass spectrometry; MVP, major vault protein; MYADM, myeloid-associated differentiation marker protein; NDRG2, N-myc downstream regulated gene 2; Neu5Ac, N-acetylneuraminic acid terminal; $\mathrm{PH}$, partial hepatectomy; $\mathrm{PHH}$, primary human hepatocytes; PRDX3, peroxiredoxin 3; SELDI-TOF-MS, surface enhanced laser desorption/ ionization time-of-flight mass spectrometry; SILAC, stable isotope labeling by amino acids in cell culture; TGM2, transglutaminase 2; TMT, tandem mass tag; TTA, tetrahydroxyxanthones.

Received: 30 October 2013; Revised: 6 December 2013; Accepted: 7 December 2013

DOI of original article: $10.14218 /$ JCTH.2013.00022.

${ }^{\S}$ Contributed equally.

* Correspondence to: Dominik A. Megger, Medizinisches Proteom-Center, RuhrUniversität Bochum, Bochum 44801, Germany, Tel: +49-234/32-26119, E-mail: dominik.megger@rub.de; Barbara Sitek, Medizinisches Proteom-Center, RuhrUniversität Bochum, Bochum 44801, Germany. Tel: +49-234/32-24362, E-mail: barbara.sitek@rub.de samples or tissue specimens are essential for the development of effective treatment strategies. Over the years, several serum marker candidates have been reported for HCC, where $\alpha$-fetoprotein (AFP) ${ }^{1}$ and des- $\gamma$-carboxyl prothrombin ${ }^{2}$ are the most-prominent and widely used ones. Regarding histopathological diagnosis of HCC, glypican-3, heat shock protein 70, and glutamine synthetase are established feasible biomarkers for HCC diagnosis using immunohistochemistry. ${ }^{3}$ However, there is still a need for further optimization, as none of the known markers exhibit $100 \%$ specificity and sensitivity, and the diagnostic performance is still insufficient for early-stage and small HCC.

To identify novel biomarker candidates, proteomic-based approaches offer a useful and versatile analytical platform, as shown by the numerous HCC studies utilizing this particular "omics"-based technology. Apart from biomarker and drug target discovery, proteome analysis is also useful for the study of the molecular processes underlying HCC progression, metastasis, and recurrence.

The current review gives a summary of the use of proteomics for the investigation of HCC. To begin, we review the general aspects of proteomics and strategies of quantitative proteome analysis. Then, we highlight selected examples of different HCC-related proteomic studies demonstrating the broad applicability of proteomics to analyze diverse sample types.

\section{Proteomic techniques}

\section{General aspects}

The proteome describes the entity of proteins that are expressed from a genome at a given time under a set of specific conditions. In contrast to the comparatively static genome, the proteome is highly dynamic due to constant alterations in expression profiles and post-translational modifications of different proteins. Thus, single genes can generate multiple protein isoforms leading to an enormous complexity of the proteome.

A typical experimental workflow of a proteomic experiment is depicted in Fig. 1. In the first step, proteins are isolated from samples originating from patients or model systems like hepatoma cell lines or HCC animal models. Depending on the scientific question, an additional fractionation step can be applied; which, for example, can enrich posttranslationally modified proteins. Subsequently, two main strategies of proteome analysis can be followed, either a topdown or a bottom-up approach. Using a top-down strategy, two-dimensional differential gel electrophoresis (2D-DIGE) is used to separate intact proteins in a gel, and proteins of interest are digested and analyzed using mass spectrometry 
Patient Samples

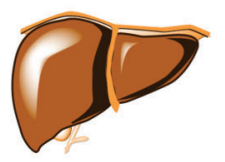

Tissues

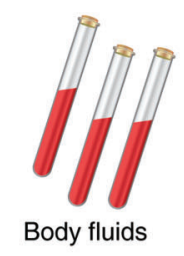

bosonswas
Model Systems

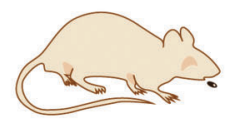

Animal models

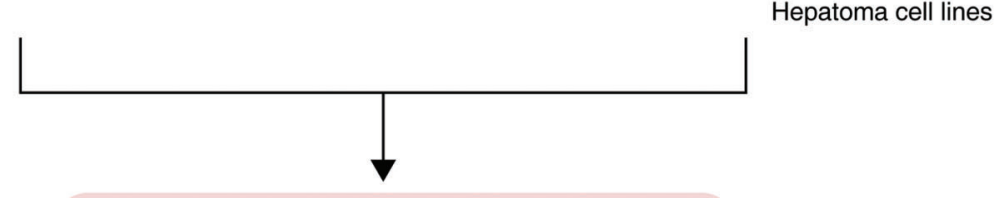

\section{Protein Isolation}

Cell lysis and solubilization of proteins Precipitation of proteins from liquid samples

\section{Top-Down Proteome Analysis}
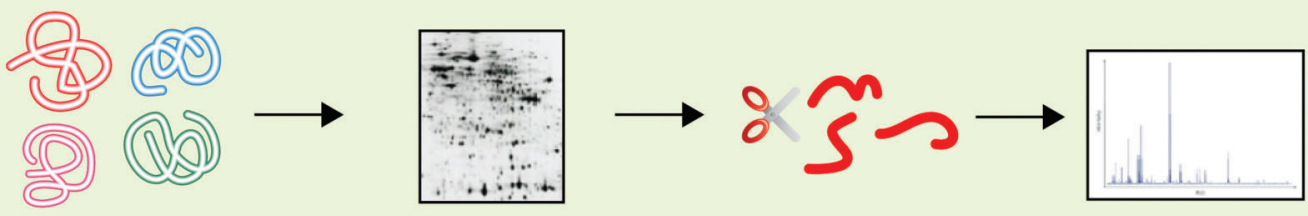

Protein mixture

2D-gel electrophoresis

Peptides of a separated protein

Mass spectrometry

\section{Bottom-Up Proteome Analysis}

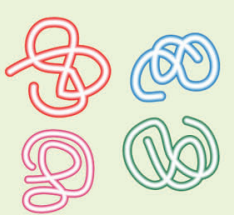

Protein mixture

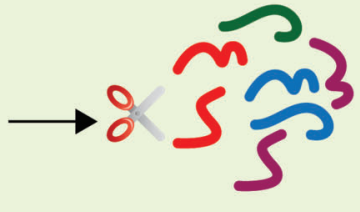

Peptides of a all proteins

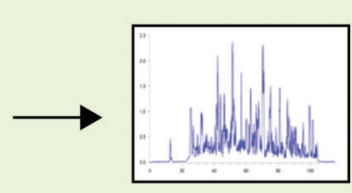

Liquid chromatography

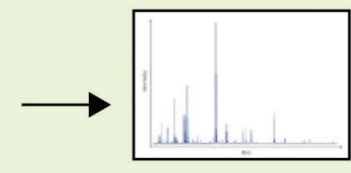

Mass spectrometry

\section{Protein Identification \\ Database search \\ De novo sequencing}

Fig. 1. A generalized workflow of a proteomics experiment showing the different principles of top-down and bottom-up proteome analyses. 
(MS). In contrast, in bottom-up experiments, protein samples are directly digested, and the generated peptides are then separated and analyzed using reversed-phase liquid chromatography coupled tandem mass spectrometry (LC-MS/MS). Irrespective of the followed strategy, proteins are finally identified by database searches or de novo sequencing.

\section{Quantification strategies}

Apart from identifying as many proteins as possible in a given sample, the accurate quantification of these biomolecules has become a major goal in the research field of proteomics. Hence, several methodologies have been developed to quantitatively monitor relative or absolute changes in protein expression. The applicability of different quantification strategies depends on the sample amount and the sample type (e.g. tissues, cultured cells, body fluids). Additionally, all quantification approaches have their intrinsic advantages and disadvantages regarding accuracy, proteome coverage, multiplexing, effort, and costs. In Fig. 2, the workflows of several widely used quantification strategies are shown, and their particular pros and cons are summarized. Although 2D-DIGE and label-free quantification by LC-MS/MS analysis have evolved into the most widely used approaches for tissuebased HCC proteome analysis, all of the mentioned strategies have been applied in proteomic studies of HCC. The following section will give an overview of various HCC proteomic studies utilizing different experimental techniques and sample types. A brief summary of selected studies is shown in Table 1.

\section{Proteomic studies of hepatocellular carcinoma}

\section{Cell culture models}

For HCC proteomic studies, HCC cell lines have been extensively studied as model systems, because of their homogeneity relative to the heterogeneous nature of tumor tissues. In addition, cell lines can be transformed, providing the opportunity to perform experiments under well-defined and manipulated conditions (e.g. knockdown or knockout of a particular gene). Furthermore, cultured cells are easily accessible and provide an endless source of protein samples. However, the main limitation of proteomic data derived from cell culture is the inherent difference between in vivo and in vitro conditions. ${ }^{4}$

Aiming for the discovery of progression biomarkers for HCC invasion, Wu et al. analyzed membrane proteins using SDS-PAGE and electrospray-ionization mass spectrometry (ESI-MS/MS) to study human HCC cell lines with different metastatic potentials. A total of 14 differentially regulated
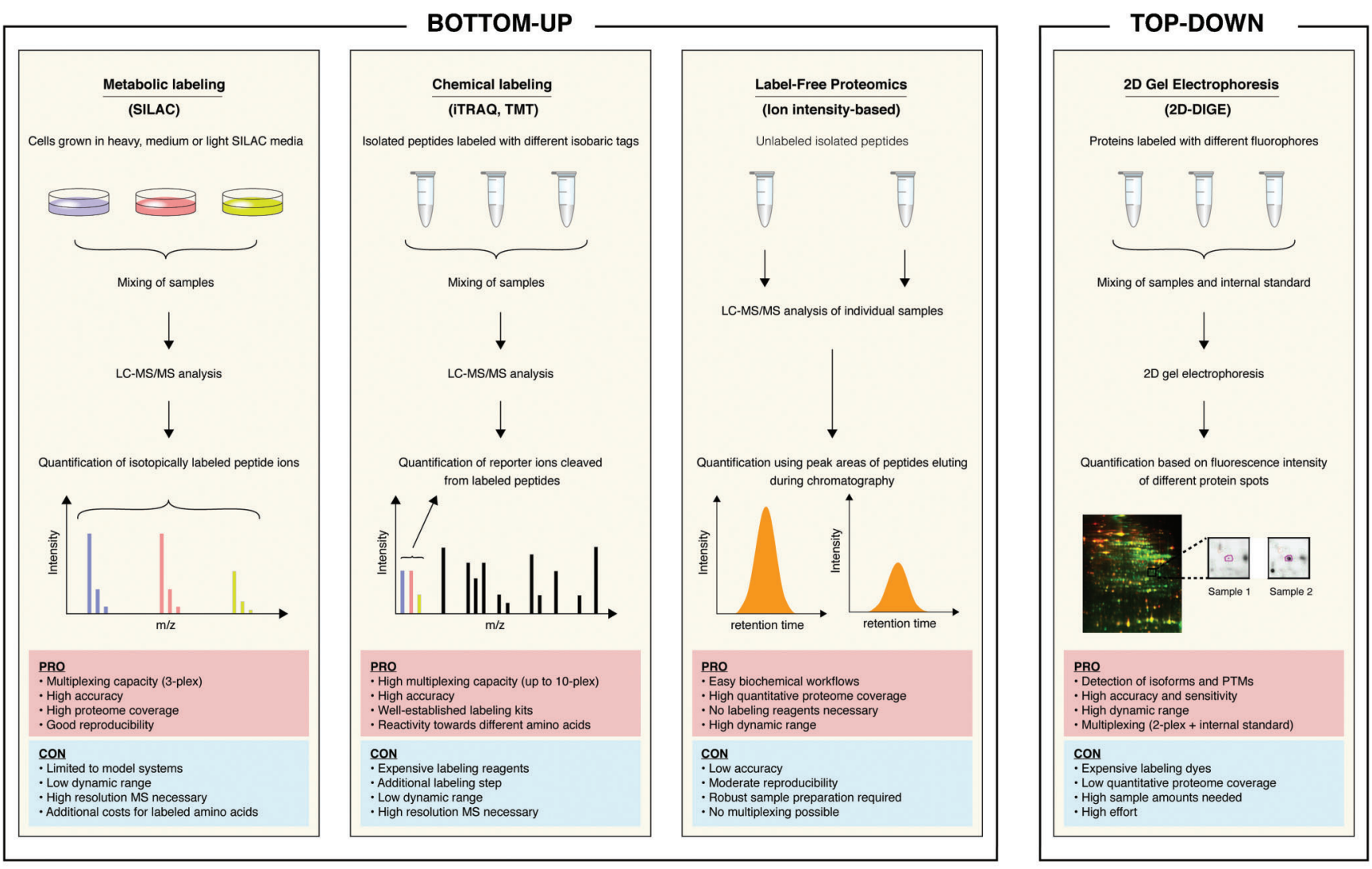

Fig. 2. Schematic representation of quantitative proteomic approaches. On the left, simplified workflows of three widely used bottom-up approaches utilizing metabolic labeling, chemical labeling, and label-free quantification are shown. On the right, a typical top-down proteomics experiment using 2D-DIGE as the quantification method is depicted. The advantages and disadvantages of the particular approaches are summarized in cyan and red boxes, respectively. Abbreviations used in this figure: SILAC, stable isotope labeling by amino acids in cell culture; iTRAQ, isobaric tags for relative and absolute quantification TMT, tandem mass tag; 2D-DIGE, two-dimensional difference gel electrophoresis. 
Megger D. A. et al: HCC proteomics

Table 1. Selected examples of proteomic studies utilizing different sample sources, quantification methods and mass-spectrometric approaches

\begin{tabular}{|c|c|c|c|c|}
\hline Sample source & Quantification & Mass spectrometry & HCC-related clinical question & Ref. \\
\hline \multirow[t]{5}{*}{ Cellular model } & SDS-PAGE & ESI-MS/MS & Biomarkers for HCC invasive progression & 5 \\
\hline & 2D-DIGE & MALDI-TOF MS & Proteomic changes related to AFP expression & 10 \\
\hline & 2D-DIGE & $\begin{array}{l}\text { MALDI-TOF MS and } \\
\text { MS/MS }\end{array}$ & Exogenous chemical effect on HCC development & 11 \\
\hline & ITRAQ & 2D LC-MS/MS & Effect of HBX protein on $\mathrm{HCC}$ angiogenesis & 12 \\
\hline & 2D-PAGE & LC-MS/MS & $\begin{array}{l}\text { Secretome analysis of primary human hepatocytes, } \\
\text { HepG2 and Hep3B cell lines }\end{array}$ & 14 \\
\hline \multirow[t]{6}{*}{ Animal models } & 2D-DIGE & MALDI-TOF MS & Effect of HBX protein on HCC development & 19 \\
\hline & Label free & LC-MS/MS & $\begin{array}{l}\text { Dysregulation of extracellular matrix proteins during } \\
\text { HCC development }\end{array}$ & 20 \\
\hline & Label free & LC-MS/MS & $\begin{array}{l}\text { Protein profile during liver regeneration after partial } \\
\text { hepatectomy }\end{array}$ & 22 \\
\hline & 2-DE & MALDI-TOF & Mechanism of induction of $\mathrm{HCC}$ by microcystins & 25 \\
\hline & 2D-DIGE & MALDI-MS & $\begin{array}{l}\text { HCC developed profiling following carcinogenic and } \\
\text { non-carcinogenic compounds }\end{array}$ & 28 \\
\hline & 2D-DIGE & MALDI TOF/TOF MS & Proteome analysis of $\mathrm{HCC}$ progression & 15 \\
\hline \multirow[t]{4}{*}{ Body fluids } & 2D-DIGE & MALDI-TOF MS/MS & HCC biomarkers in serum & 31 \\
\hline & Label free & LC-MS/MS & HCC biomarkers in serum & 33 \\
\hline & Label free & LC-ESI-MS/MS & Glycoproteomics changes associated with $\mathrm{HCC}$ in sera & 34 \\
\hline & MRM & LC-ESI-MS/MS & HCC biomarkers in serum & 35 \\
\hline \multirow[t]{7}{*}{$\begin{array}{l}\text { Human tissue } \\
\text { samples }\end{array}$} & $\begin{array}{l}\text { Label free and } \\
\text { 2D-DIGE }\end{array}$ & $\begin{array}{l}\text { LC-MS/MS and } \\
\text { MALDI-TOF MS }\end{array}$ & Diagnostic HCC biomarkers & 36 \\
\hline & ITRAQ & LC-MS \MS & Diagnostic HCC biomarkers & 41 \\
\hline & 2D-DIGE & MALDI-TOF MS & $\begin{array}{l}\text { Hepatocarcinogenesis mechanism following liver } \\
\text { cirrhosis and chronic hepatitis virus B infection }\end{array}$ & 37 \\
\hline & CICAT & 2D-LC-MS/MS & Prognostic biomarkers for HCC & 42 \\
\hline & Label free & LC-MS/MS & $\begin{array}{l}\text { Prognostic biomarkers for HCC related to telomerase } \\
\text { reverse transcriptase (hTERT) }\end{array}$ & 43 \\
\hline & 2D-DIGE & LC-MS/MS & HCC progression and prognosis & 44 \\
\hline & 2D-DIGE & MALDI-TOF MS & HCC progression & 45 \\
\hline
\end{tabular}

proteins were identified in the HCC cell lines with high (HCCLM9) and low (MHCC97L) metastatic potential. Using immunohistochemistry, over-expression of the protein Coronin-1C was successfully verified in clinical HCC tumor tissue samples with metastasis and invasive behavior. ${ }^{5}$ This study and others ${ }^{6-9}$ demonstrated the applicability of the cell culture model in biomarker discovery studies.

Taking the advantage of the homogeneity of cell culture models, Yokoo et al. analyzed the differences in protein expression between nine AFP-producing cell lines and seven AFP-negative cell lines using 2D-DIGE and matrix-assisted laser desorption-ionization time-of-flight mass spectrometry (MALDI-TOF MS). Of 2000 quantified proteins, 11 proteins were identified and reported to be differentially expressed between these two cell lines, most of which were mainly involved in glucose metabolism, apoptosis, cytoskeleton, and translation. ${ }^{10}$

Another advantage of using cell culture models is the ability to monitor the effect of exogenous or endogenous chemicals on protein expression and associated pathways. Recently, Fu et al. used 2D-DIGE and MALDI-TOF MS and MS/ MS to identify 1,3,6,7-tetrahydroxyxanthones' (TTA) targets in $\mathrm{HCC}$ cells. In total, 18 proteins were differentially regulated in treated and non-treated cell lines. Of interest, $\beta$-tubulin and translationally controlled tumor proteins were downregulated, whereas $14-3-3 \sigma$ and P16 proteins were upregulated. ${ }^{11}$

Cell culture models can provide informative data regarding the effects of changing expression of a specific enzyme on other proteins or whole pathways. This method was applied by Zhang et al. ${ }^{12}$ to study the effect of hepatitis $B$ virus $X$ protein $(\mathrm{HBX})$ on protein expression correlated with angiogenesis in HCC cell lines. ${ }^{12}$ Using isobaric tags for relative and absolute quantitation (iTRAQ) labeling for quantification, 16 proteins were reported to be differently expressed between HepG2 cells transfected with HBV genome and empty vector as control. In particular, fumarate hydratase and tryptophan-tRNA ligase, which were previously described in association with angiogenesis, were upregulated in HBV transfected cells in this study.

Secreted proteins play important roles in cell migration, communication, and signaling. ${ }^{13}$ Thus the cancer cell secretome has been studied as a potential source of HCC biomarkers. Slany et al. applied LC-MS/MS analysis on isolated 2D-PAGE spots of primary human hepatocytes $(\mathrm{PHH}), \mathrm{HepG} 2$, and Hep3B cells' secretome. ${ }^{14}$ An overlap was reported between the secretome and plasma proteins in all three cell lines. Out of 72 identified proteins in the 
secretome of $\mathrm{PHH}, 46$ were reported in plasma as well. For HepG2 and Hep3B secretomes, 55 and 24 plasma proteins were detected, respectively. Among the identified secreted proteins, many were already reported in association with HCC, including ephrin-A1, neuroserpin, glypican-3, and gastricsin. In addition, since stanniocalcin-2 precursor and bactericidal/permeability-increasing protein-like 1 demonstrated a restricted expression profile in HepG2 secretome, they were identified as novel potential HCC biomarkers. ${ }^{14}$

\section{Animal models}

$\mathrm{HCC}$ is a multifactorial disease, and ideally studied under controlled environmental conditions where data regarding disease progression, management, and cause can be more reliably generated. Animal HCC models are well characterized and easy to handle, making them an important source for in vivo proteomic data. However, induction of HCC in animals takes place over a short period of time, while HCC in humans normally develops over years. Consequently, not all HCCrelevant proteome changes in humans can be reflected in animal models. ${ }^{15}$

$\mathrm{HBV}$ is a risk factor for $\mathrm{HCC}$ by producing $\mathrm{HBx}$, a protein implicated in HCC development. ${ }^{16-18}$ In order to further understand the relationship between $\mathrm{HBx}$ and HCC, a proteomic study comparing liver tissue from $\mathrm{HBx}$ transgenic and control mice has been performed. Here, seven proteins were found by 2D-DIGE and MALDI-TOF MS to be upregulated in tumor tissue of the $\mathrm{p} 21-\mathrm{HBx}$ transgenic relative to control mice. The over expression of PSMB4, CTSB, PSMA1, and PSMC3 in HCC was confirmed using RT-PCR in tumor transgenic mice and human liver tissue. ${ }^{19}$

Lai et al. ${ }^{20}$ were the first to apply proteomics to compare changes in extracellular matrix (ECM) proteins during the development of HCC from fibrosis and steatosis. They used liver tissue sample obtained from two transgenic mouse models of disease progression, PDGFC transgenic and Pten null mice. Using multi-dimensional protein separation, 10,707 protein isoforms were identified following 2D-HPLC and SDS-PAGE for extensive protein separation and nanoflow LC-MS/MS analysis for protein identification. Twenty six collagen proteins demonstrated a significant change during HCC progression, especially collagen types IV, VI, XIV, XV, and XVI. For non-collagenous proteins in ECM, laminin 521 protein was the most abundant laminin protein in HCC, and the receptor protein Integrin $\alpha 3$ was reported only in the Pten null mouse tumor. ${ }^{20}$

Proteome analysis of animal models can also facilitate HCC diagnosis and implementation of appropriate therapeutic strategies. As partial hepatectomy $(\mathrm{PH})$ is a first line therapy for a certain population of HCC patients, ${ }^{21}$ Cao et al. performed proteomic analysis in mouse models with $50 \%$ $\mathrm{PH}$ in order to detect changes in protein expression profiles during liver regeneration. ${ }^{22}$ Using a two-dimensional set-up, including SDS-PAGE and LC-MS/MS analysis, an average of 441 differentially regulated proteins in mouse liver tissue of sham-operated control and the $50 \% \mathrm{PH}$ group were identified. One day after $\mathrm{PH}, 87$ protein expression profiles were changed; where 50 proteins were upregulated, and 37 proteins were downregulated in the $50 \% \mathrm{PH}$ group. All proteins were related to critical biological pathways.

Microcystins (MCs) are known inducers of HCC. ${ }^{23-24}$ In order to understand the carcinogenic processes underlying induction of HCC by MCs, a proteomic analysis was performed in animal models. MicroRNAs and protein expression changes were monitored in mouse liver treated with microcystins-LR. Using two-dimensional gel electrophoresis, 62 proteins were reported to be significantly regulated in microcystins-LR treated mice relative to untreated controls. MALDI-TOF was used to identify 42 proteins. From the identified proteins, two tumorigenesis associated proteins, transglutaminase 2 (TGM2) and $\mathrm{N}$-myc downstream regulated gene 2 (NDRG2) were verified using Western blot analysis. ${ }^{25}$

Chu et al. investigated the proteins involved in peroxisome proliferation using surface enhanced laser desorption/ionization time-of-flight mass spectrometry (SELDI-TOF-MS) to compare protein profiles of control (wild type mice), acyl-CoA oxidase-deficient mice (AOX-/-), and peroxisome proliferator (Wy-14,643) wild type-treated mice. ${ }^{26}$ 2D-DIGE and MALDIMS analyses were used to compare the protein regulation profiles of HCC in rat liver tissue induced by carcinogenesis and non-carcinogenesis compounds. Zeindl-Eberhart et al. reported aldose reductase-like proteins (ARLPs) 1-4 to be expressed higher in both HCC induced models relative to normal rat liver. ${ }^{27}$ Perhaps, as suggested by the authors, ARLPs are viable immunohistochemical diagnostic biomarkers of HCC in humans. ${ }^{28}$

In order to characterize proteome changes during liver cancer progression, tissue proteomics samples from rats of six progression stages of liver cancer were analyzed by 2DDIGE and MALDI TOF/TOF MS. ${ }^{15}$ Interestingly, most of the differentially expressed proteins in malignant stages were altered in the pre-tumorous phase. This finding could aid in the identification of reliable biomarker candidates for the early diagnosis for HCC.

\section{Body fluids}

Despite its low sensitivity of $39 \%-65 \%$, AFP is the only commonly used diagnostic serum biomarker for HCC. ${ }^{29}$ This highlights an urgent need for the identification of new HCC serum markers with higher sensitivities and specificities. Plasma contains thousands of proteins that could potentially reflect the pathological and physiological status of patients, such as aberrant secretions and tissue leakage proteins. Plasma proteins distribute over a wide dynamic quantitative range. For example, albumin is found from $35-40 \mathrm{mg} / \mathrm{ml}$, and interleukin 6 is found from $0-5 \mathrm{pg} / \mathrm{ml}$. In most cases, potential biomarkers are present at low concentrations; however, more than $99 \%$ of the entire protein amount of serum is represented by only 22 proteins. That makes proteomics analysis of plasma a very challenging task, even with available advanced analytical technologies such as 2DDIGE and LC-MS/MS. ${ }^{30}$

In biomarker studies, high abundant proteins are mostly removed by immunodepletion in order to enrich remaining low abundant serum proteins. Feng et al. depleted albumin and immunoglobulin IgG from the sera of $20 \mathrm{HBV}$ patients, 20 HCC patients, and 20 healthy controls to identify potential HCC biomarkers in serum. Proteins were analyzed by 2DDIGE, and out of 40 differentially expressed proteins, eight proteins were identified with MALDI-TOF MS/MS. Heat-shock protein 27 (HSP 27) was verified as a potential biomarker in sera by Western blotting. ${ }^{31}$ The use of more extensive sample preparation workflow can be followed to discover novel HCC biomarkers in serum, including immunodepletion of the six most abundant proteins (transferrin, albumin, antitrypsin, IgG, IgA, and haptoglobin), fluorophore labeling, and 3D 
protein separation, including online 1D electrophoresis after anion exchange and reversed phase LC. ${ }^{32}$

In a more recent study investigating the discovery of novel HCC markers in plasma, ${ }^{33}$ immunodepletion of IgG and albumin was applied to nine pooled plasma samples from 120 controls, 99 liver cirrhosis patients, and 120 HCC patients. In order to decrease proteome complexity, each group of pooled sample was further fractionated to yield 24 fractions, and only seven were chosen for label free analysis and LC-MS/MS. In total, 1,307 proteins were identified, and 26 proteins were found to be differentially regulated among the experimental groups. Four potential HCC biomarker candidates (apolipoprotein A1, complement component 3, alpha-1-antitrypsinand hemopexin) were subsequently verified using ELISA. The expression trends of these proteins were confirmed in an independent patient cohort as well.

Despite being widely used, depletion of high abundant proteins from plasma or serum is a controversial issue in proteomics. This is due to its insufficient reproducibility and the ability of some high abundant proteins, such as albumin, to bind other proteins that are then unintentionally codepleted. If such proteins are completely removed by this co-depletion, information regarding alterations in their expression would be lost. If proteins were only partially removed, misleading information regarding artificially generated changes in protein levels that are not associated with the disease would be detected. For example, Feng et at. nicely demonstrated this problem when they showed that $\alpha$ fetoprotein was non-specifically depleted with the albuminIgG fraction. ${ }^{31}$

In addition to depletion techniques, enrichment of proteins in plasma and serum are also widely used in proteomic studies. For example, Yang et al. used the Con A-magnetic particle conjugate-based method to isolate glycoproteins from the sera of 60 healthy controls and $60 \mathrm{HCC}$ patients to study the difference in glycoproteomics associated with HCC. Ninety-three glycoproteins were identified by LC-ESI-MS/MS in the controls compared to 85 in HCC patients' sera, and 72 glycoproteins overlapped between the two samples sets. In addition, the authors investigated the different $N$-glycan characteristics linked to HCC by MALDI-TOF/MS. ${ }^{34} \mathrm{~N}$-glycans in HCC were reported to be hyperfucosylated and associated with a reduction of $\mathrm{N}$-acetylneuraminic acid terminal (Neu5Ac).

In the past few years, targeted mass spectrometry-based approaches like multiple reaction monitoring (MRM) have become more widely used in proteomic studies of body fluids. However, such targeted methods are not applicable for untargeted discovery studies. Hence, prior identification of protein or peptide targets using other proteomic techniques is required. Using isotopically labeled forms of this target that are spiked into a given sample, the MRM approach can be used to absolutely quantify the target protein in a complex of non-depleted matrix. In a recent study, MRM was used to verify nominated HCC diagnostic proteins in plasma from global data mining of proteins linked to HCC. Of the 4,658 proteins screened in an extensive data filtration process, nine proteins were found to be differentially expressed using MRM for quantification in the plasma of $18 \mathrm{HCC}$ patients before and after a three month treatment regimen and 36 healthy controls. Compared with AFP alone, a multimarker panel of Actin-binding protein anillin (ANLN) and filamin-B (FLNB) was reported to discriminate the healthy controls from the HCC group before treatment. ${ }^{35}$

\section{Human tissue samples}

An extensive proteome analysis of clinical tissue samples is the most common source of HCC proteomic data, where most researchers apply comprehensive proteomic analysis on HCC tumor and adjacent non-tumor tissue to address different clinical questions (Fig. 3). ${ }^{32,36-40}$

Very recently, our group used two different proteomic approaches to characterize protein changes associated with $\mathrm{HCC}$ in tissue and to identify novel biomarker candidates. ${ }^{36}$ In a comprehensive proteomic analysis, seven HCC and adjacent non-tumorous liver tissue samples were quantitatively analyzed by 2D-DIGE and label-free LC-MS/MS. As a result, we identified 476 and 148 proteins that were differentially expressed between normal and HCC tissue in the label-free and 2D-DIGE analysis, respectively. Following verification using Western blotting, two of six selected candidates, major vault protein (MVP) and betaine-homocysteine S-methyltransferase 1 (BHMT), were verified in an independent and larger patient cohort.

Chemical labeling strategies, such as iTRAQ-based quantitative proteome analysis, can be used to identify differentially regulated proteins in $\mathrm{HCC}$ tissue relative to adjacent non-tumorous controls. Using this technique, over 600 proteins were identified in a LC-MS/MS analysis by Chaerkady et al. ${ }^{41}$ Based on alterations in the iTRAQ reporter ion intensities, 59 proteins were found to be upregulated, and 92 proteins were downregulated in HCC. Interestingly, myeloid-associated differentiation marker protein (MYADM) showed $100 \%$ specificity and $63 \%$ sensitivity in a subsequent verification screen using tissue microarray consisting of 53 HCC tissue and 20 non-tumourous liver tissue samples. ${ }^{41}$

To provide further insight into the mechanism underlying hepatocarcinogenesis, $\mathrm{Li}$ et al. carried out a differential proteomic analysis on liver cirrhosis (LC) and hepatitis B virus (CHB)-developed HCC and their adjacent non-tumorous liver tissues. Twelve LC-developed HCC and six CHB-developed $\mathrm{HCC}$ tissue samples and adjacent non-tumorous liver tissue

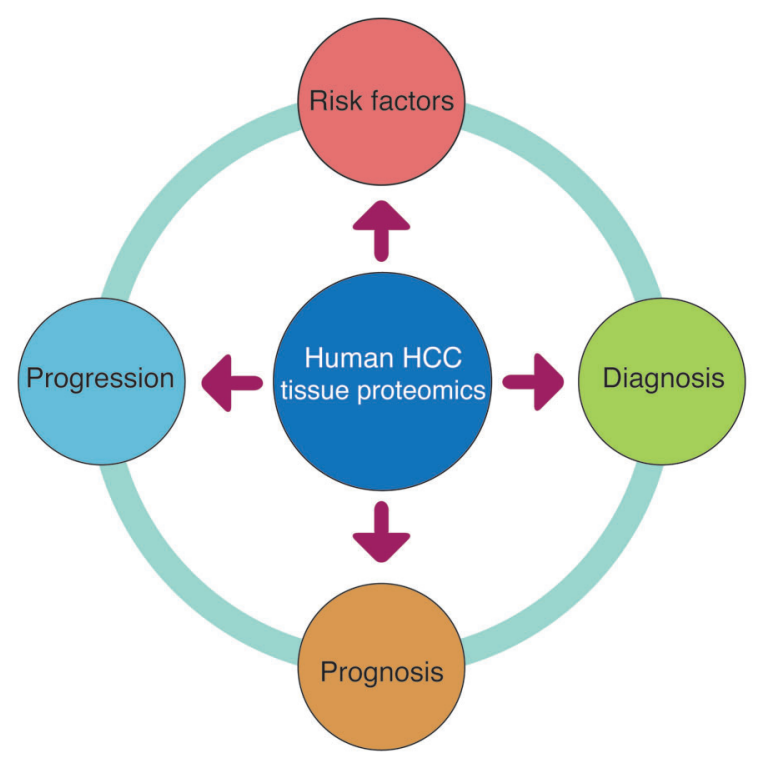

Fig. 3. HCC-related questions mostly pursued in tissue-based proteomic studies using human HCC samples. 
were analyzed using 2D-DIGE and MALDI-TOF MS. Seventeen proteins were differentially regulated between the cancerous and non-cancerous tissue. In particular, ADP/ATP carrier protein and c-Jun $\mathrm{N}$-terminal kinase 2 were reported to be upregulated only in CHB-developed HCC samples, while RhoGTPase-activating protein 4 was downregulated. In contrast, insulin-like growth factor binding protein 2 was downregulated in LC-developed HCC. ${ }^{37}$

Bai et al. searched for prognostic markers in $252 \mathrm{HCC}$ patients who underwent liver transplantation (LT) treatment. During a median follow-up of 42 months, 79 patients demonstrated recurrence. Tissue laser capture microdissection (LCM) was performed to isolate HCC hepatocytes from liver tissue samples and cleavable isotope-coded affinity tags (CICAT) were used to quantitate changes in protein expression in a 2D-LC-MS/MS analysis. From 52 differentially expressed proteins in recurrence and no-recurrence after LT, calpain small subunit 1 (CAPN4) overexpression was correlated with $\mathrm{HCC}$ recurrence and metastasis, making it a potential candidate for poor HCC prognosis. ${ }^{42}$

More recently, Mizuno et al. searched for novel HCC prognostic proteins related to human telomerase reverse transcriptase (hTERT). A specific proteomic approach was used that included fractionation of protein lysates using gel filtration columns to isolate hTERT proteins and analysis with LC-MS/MS. The expression of hnRNP A2/B1 protein was reported to be a potential marker for HCC survival prediction following surgical resection. ${ }^{43}$

Proteomic profiling of HCC tissue with different histological differentiation was conducted by Orimo et al. to monitor key proteins involved in HCC progression. They analyzed microdissected cells from six well differentiated, 14 moderately differentiated, and seven poorly differentiated HCC tissue, in addition to 11 adjacent non-tumorous liver tissue and seven normal liver tissue. Proteins were profiled using 2D-DIGE, and 21 proteins from 41 selected 2D-DIGE spots were identified using LC-MS/MS. APC-binding protein EB1 (EB1) was a nominated potentially important protein for HCC. Thus, EB1 was further tested in an additional 145 HCC samples using immunohistochemistry. Interestingly, EB1 was reported only in moderate and poor differentiated HCC tissue, and its expression was associated with poor HCC prognosis. ${ }^{44}$

In another study characterizing proteome changes associated with HCC progression in order to establish new therapeutic strategies for HCC, 2D-DIGE with MALDI-TOF MS were used to profile protein expression changes in $27 \mathrm{HCC}$ tissue samples with different histological backgrounds and their adjacent non-tumorous liver tissue. From 43 selected spots, 22 differentially expressed proteins were identified following MS analysis. Of these proteins, peroxiredoxin 3 (PRDX3) was reported to be overexpressed in poorly differentiated HCC tissue, and it may have a role in HCC progression. $^{45}$

\section{Conclusions}

In this review we have presented several fundamental aspects of proteomics and different quantitative methodologies applicable to identify protein alterations associated with HCC. By highlighting selected examples from the literature, we illustrated the broad range of potential applications of proteomics in HCC research. As a final remark, we want to emphasize that proteomics is a powerful and versatile bioanalytical technique. However, when using this platform one should always keep in mind the potential pitfalls and error sources present in almost every proteomic workflow in order to obtain reliable results that are indeed disease-related and not merely experimental artifacts. Therefore, researchers need to identify whether the chosen study design is applicable to pursue a particular scientific/clinical question and if the size of the sample cohort is sufficient to reach a statistical significance. Furthermore, the single steps within the followed proteomic workflow need to be reproducible, robust, and reliable. Thus, techniques like enrichment or depletion should be avoided if possible. When using human tissue samples several other aspects need to be considered. These include the quality and homogeneity of the sample, which can be significantly improved by utilizing microdissection. Additionally, detailed information regarding the patients such as underlying disease, ethnic group, TNM staging, therapy/ treatment, and follow-up status should ideally be known, as the consideration of such additional information can clearly improve the quality of a proteomic study. To meet all of the aforementioned demands, intense collaborations between clinicians, pathologists, and bioanalytical researchers are always preferable to ensure high quality proteomic research.

\section{Acknowledgements}

D. A. Megger and B. Sitek acknowledge the financial support from the PROFILE-consortium Ruhr. PROFILE is co-funded by the European Union (European Regional Development Fund Investing in your future) and the German federal state North Rhine-Westphalia (NRW), project number z0911bt004e. Furthermore, partial funding from P.U.R.E. (Protein Unit for Research in Europe), a project of North Rhine-Westphalia as well, is acknowledged.

\section{Conflict of interest}

The authors declare no conflicts of interest.

\section{Author contributions}

Writing the manuscript, designing the figures and performing literature searches (DAM, WN), further contribution in writing of the manuscript (BS and HEM).

\section{References}

[1] Di Bisceglie AM, Sterling RK, Chung RT, Everhart JE, Dienstag JL, Bonkovsky $\mathrm{HL}$, et al. Serum alpha-fetoprotein levels in patients with advanced hepatitis C: results from the HALT-C Trial. J Hepatol 2005;43:434-441.

[2] Liebman HA, Furie BC, Tong MJ, Blanchard RA, Lo KJ, Lee SD, et al. Des- $\gamma$ Carboxy (abnormal) prothrombin as a serum marker of primary hepatocellular carcinoma. N Engl J Med 1984;310:1427-1431.

[3] Di Tommaso L, Franchi G, Park YN, Fiamengo B, Destro A, Morenghi E, et al. Diagnostic value of HSP70, glypican 3 , and glutamine synthetase in hepatocellular nodules in cirrhosis. Hepatology 2007;45:725-734.

[4] Tan HT, Lee YH, Chung MC. Cancer proteomics. Mass Spectrom Rev 2012;31: 583-605.

[5] Wu L, Peng CW, Hou JX, Zhang YH, Chen C, Chen LD, et al. Coronin-1C is a novel biomarker for hepatocellular carcinoma invasive progression identified by proteomics analysis and clinical validation. J Exp Clin Cancer Res 2010; 29:17.

[6] Ding SJ, Li Y, Tan YX, Jiang MR, Tian B, Liu YK, et al. From proteomic analysis to clinical significance: overexpression of cytokeratin 19 correlates with hepatocellular carcinoma metastasis. Mol Cell Proteomics 2004;3: $73-81$. 
[7] Li Y, Tang ZY, Tian B, Ye SL, Qin LX, Xue O, et al. Serum CYFRA 21-1 level reflects hepatocellular carcinoma metastasis: study in nude mice model and clinical patients. J Cancer Res Clin Oncol 2006;132:515-520.

[8] Chen N, Sun W, Deng X, Hao Y, Chen X, Xing B, et al. Quantitative proteome analysis of HCC cell lines with different metastatic potentials by SILAC. Proteomics 2008;8:5108-5118.

[9] Fujii K, Kondo T, Yokoo H, Yamada T, Iwatsuki K, Hirohashi S. Proteomic study of human hepatocellular carcinoma using two-dimensional difference gel electrophoresis with saturation cysteine dye. Proteomics 2005;5:14111422.

[10] Yokoo H, Kondo T, Fujii K, Yamada T, Todo S, Hirohashi S. Proteomic signature corresponding to alpha fetoprotein expression in liver cancer cells. Hepatology 2004;40:609-617.

[11] Fu WM, Zhang JF, Wang H, Tan HS, Wang WM, Chen SC, et al. Apoptosis induced by $1,3,6,7$-tetrahydroxyxanthone in Hepatocellular carcinoma and proteomic analysis. Apoptosis 2012;17:842-851.

[12] Zhang J, Niu D, Sui J, Ching CB, Chen WN. Protein profile in hepatitis B virus replicating rat primary hepatocytes and HepG2 cells by iTRAQ-coupled 2-D LC-MS/MS analysis: Insights on liver angiogenesis. Proteomics 2009;9: 2836-2845.

[13] Makridakis M, Vlahou A. Secretome proteomics for discovery of cancer biomarkers. J Proteomics 2010;73:2291-2305.

[14] Slany A, Haudek VJ, Zwickl H, Gundacker NC, Grusch M, Weiss TS, et al. Cell characterization by proteome profiling applied to primary hepatocytes and hepatocyte cell lines Hep-G2 and Hep-3B. J Proteome Res 2009;9:6-21.

[15] Albrethsen J, Miller LM, Novikoff PM, Angeletti RH. Gel-based proteomics of liver cancer progression in rat. Biochim Biophys Acta 2011;1814:13671376.

[16] Wang F, Zhou H, Yang Y, Xia X, Sun Q, Luo J, et al. Hepatitis B virus X protein promotes the growth of hepatocellular carcinoma by modulation of the Notch signaling pathway. Oncol Rep 2012;27:1170-1176.

[17] Yen CJ, Lin YJ, Yen CS, Tsai HW, Tsai TF, Chang KY, et al. Hepatitis B virus X protein upregulates mTOR signaling through IKKbeta to increase cell proliferation and VEGF production in hepatocellular carcinoma. PLoS One 2012; 7:e41931.

[18] Tanaka Y, Kanai F, Ichimura T, Tateishi K, Asaoka Y, Guleng B, et al. The hepatitis $B$ virus $X$ protein enhances AP-1 activation through interaction with Jab1. Oncogene 2005;25:633-642.

[19] Cui F, Wang Y, Wang J, Wei K, Hu J, Liu F, et al. The up-regulation of proteasome subunits and lysosomal proteases in hepatocellular carcinomas of the HBx gene knockin transgenic mice. Proteomics 2006;6:498-504.

[20] Lai KK, Shang S, Lohia N, Booth GC, Masse D], Fausto N, et al. Extracellular matrix dynamics in hepatocarcinogenesis: a comparative proteomics study of PDGFC transgenic and Pten null mouse models. PLoS Genet 2011;7: e1002147.

[21] Nguyen KT, Gamblin TC, Geller DA. World review of laparoscopic liver resection-2,804 patients. Ann Surg 2009;250:831-841.

[22] Cao H, Yu J, Xu W, Jia X, Yang J, Pan Q, et al. Proteomic analysis of regenerating mouse liver following $50 \%$ partial hepatectomy. Proteome Sci $2009 ; 7: 48$.

[23] Ito E, Kondo F, Terao K, Harada K. Neoplastic nodular formation in mouse liver induced by repeated intraperitoneal injections of microcystin-LR. Toxicon 1997; 35:1453-1457.

[24] Ueno Y, Nagata S, Tsutsumi T, Hasegawa A, Watanabe MF, Park HD, et al. Detection of microcystins, a blue-green algal hepatotoxin, in drinking water sampled in Haimen and Fusui, endemic areas of primary liver cancer in China, by highly sensitive immunoassay. Carcinogenesis 1996;17:13171321.

[25] Zhao Y, Xie P, Fan H. Genomic profiling of microRNAs and proteomics reveals an early molecular alteration associated with tumorigenesis induced by MC-LR in mice. Environ Sci Technol 2011;46:34-41.

[26] Chu R, Zhang W, Lim H, Yeldandi AV, Herring C, Brumfield L, et al. Profiling of acyl-CoA oxidase-deficient and peroxisome proliferator Wy14,643-treated mouse liver protein by surface-enhanced laser desorption/ionization ProteinChip Biology System. Gene Expr 2002;10:165-177.

[27] Zeindl-Eberhart E, Klugbauer S, Dimitrijevic N, Jungblut PR, Lamer S, Rabes HM. Proteome analysis of rat hepatomas: carcinogen-dependent tumorassociated protein variants. Electrophoresis 2001;22:3009-3018.

[28] Zeindl-Eberhart E, Haraida S, Liebmann S, Jungblut PR, Lamer S, Mayer D, et al. Detection and identification of tumor-associated protein variants in human hepatocellular carcinomas. Hepatology 2004;39:540-549.

[29] Daniele B, Bencivenga A, Megna AS, Tinessa V. Alpha-fetoprotein and ultrasonography screening for hepatocellular carcinoma. Gastroenterology 2004;127:S108-S112.

[30] Anderson NL, Anderson NG. The human plasma proteome: history, character, and diagnostic prospects. Mol Cell Proteomics 2002;1:845-867.

[31] Feng JT, Liu YK, Song HY, Dai Z, Qin LX, Almofti MR, et al. Heat-shock protein 27: a potential biomarker for hepatocellular carcinoma identified by serum proteome analysis. Proteomics 2005;5:4581-4588.

[32] Feng JT, Shang S, Beretta L. Proteomics for the early detection and treatment of hepatocellular carcinoma. Oncogene 2006;25:3810-3817.

[33] Fye HK, Wright-Drakesmith C, Kramer HB, Camey S, da Costa AN, Jeng A, et al. Protein profiling in hepatocellular carcinoma by label-free quantitative proteomics in two west african populations. PLoS One 2013;8:e68381.

[34] Yang G, Cui T, Wang Y, Sun S, Ma T, Wang T, et al. Selective isolation and analysis of glycoprotein fractions and their glycomes from hepatocellular carcinoma sera. Proteomics 2013;13:1481-1498.

[35] Kim H, Kim K, Yu SJ, Jang ES, Yu J, Cho G, et al. Development of biomarkers for screening hepatocellular carcinoma using global data mining and multiple reaction monitoring. PLoS One 2013;8:e63468.

[36] Megger DA, Bracht T, Kohl M, Ahrens M, Naboulsi W, Weber F, et al. Proteomic Differences Between Hepatocellular Carcinoma and Nontumorous Liver Tissue Investigated by a Combined Gel-based and Label-free Quantitative Proteomics Study. Mol Cell Proteomics 2013;12:2006-2020.

[37] Li N, Long Y, Fan X, Liu H, Li C, Chen L, et al. Proteomic analysis of differentially expressed proteins in hepatitis $B$ virus-related hepatocellular carcinoma tissues. J Exp Clin Cancer Res 2009;28:122.

[38] Li C, Ruan HQ, Liu YS, Xu MJ, Dai J, Sheng QH, et al. Quantitative proteomics reveal up-regulated protein expression of the SET complex associated with hepatocellular carcinoma. J Proteome Res 2012;11:871-885.

[39] Liang CR, Leow CK, Neo JC, Tan GS, Lo SL, Lim JW, et al. Proteome analysis of human hepatocellular carcinoma tissues by two-dimensional difference gel electrophoresis and mass spectrometry. Proteomics 2005;5:2258-2271.

[40] Luk JM, Lam CT, Siu AF, Lam BY, Ng IO, Hu MY, et al. Proteomic profiling of hepatocellular carcinoma in Chinese cohort reveals heat-shock proteins (Hsp27, Hsp70, GRP78) up-regulation and their associated prognostic values. Proteomics 2006;6:1049-1057.

[41] Chaerkady R, Harsha HC, Nalli A, Gucek M, Vivekanandan P, Akhtar J, et al. A quantitative proteomic approach for identification of potential biomarkers in hepatocellular carcinoma. J Proteome Res 2008;7:4289-4298.

[42] Bai DS, Dai Z, Zhou J, Liu YK, Qiu SJ, Tan CJ, et al. Capn4 overexpression underlies tumor invasion and metastasis after liver transplantation for hepatocellular carcinoma. Hepatology 2009;49:460-470.

[43] Mizuno H, Honda M, Shirasaki T, Yamashita T, Yamashita T, Mizukoshi E, et al. Heterogeneous nuclear ribonucleoprotein A2/B1 in association with hTERT is a potential biomarker for hepatocellular carcinoma. Liver Int 2012;32:11461155.

[44] Orimo T, Ojima H, Hiraoka N, Saito S, Kosuge T, Kakisaka T, et al. Proteomic profiling reveals the prognostic value of adenomatous polyposis coli-endbinding protein 1 in hepatocellular carcinoma. Hepatology 2008;48:18511863.

[45] Qiao B, Wang J, Xie J, Niu Y, Ye S, Wan Q, et al. Detection and identification of peroxiredoxin 3 as a biomarker in hepatocellular carcinoma by a proteomic approach. Int J Mol Med 2012;29:832-840. 